\title{
Towards on-chip tunable nanolasers based on optomechanical zipper cavities
}

\author{
Raviv Perahia, Thiago P. Mayer Alegre, Justin D. Cohen and Oskar Painter \\ Department of Applied Physics, California Institute of Technology, Pasadena, CA 91125, USA. \\ e-mail: rperahia@caltech.edu
}

\begin{abstract}
Work towards semiconductor nanolasers at $\lambda=1.3 \mu \mathrm{m}$ in optomechanically coupled one dimensional photonic-crystal cavities is presented. Optical mode spectroscopy and on-chip tuning capability based on capacitive actuation is developed. Experimental and theoretical results are presented. (C) 2009 Optical Society of America

OCIS codes: (250.5960) Semiconductor lasers; (120.4880) Optomechanics
\end{abstract}

As the dimensions of semiconductor lasers are reduced to the nanoscale, new possibilities for studying fundamental device physics [1] and synergetic integration with other nanoscale technologies arise. Recent progress in the field of nanoscale lasers has included the incorporation of metal layers for localization and guiding of light through coupling to surface-plasmon-polariton excitations [2, 3, 4]. In conjunction with the new developments in nanolaser cavity geometries, there has been the continued interest in, and need for, methods of extending both tuning bandwidth and tuning speed beyond traditional thermal and current injection based tuning mechanisms [5, 6]. Several mechanisms have been proposed and demonstrated based on optofluidics [7] and micro-electro-mechanical-systems (MEMS) [8, 9]. We present work towards lasing and on-chip tuning of nanolasers at $\lambda=1.3 \mu \mathrm{m}$ based on optomechanically coupled one dimensional photonic-crystal cavities ("zipper" cavities) [10].

Zipper cavities are comprised of two doubly clamped nanobeams patterned with a photonic crystal structure (Fig. 1(a)) and have a highly dispersive dependence on the gap between nanobeams. The mechanical response can be tailored to be anywhere from $\mathrm{kHz}$ to $\mathrm{GHz}$ by engineering the structure's mass and spring constant. Optical defect modes are formed by a parabolic variation of the lattice constant at the center of the cavity. Using finite-element-method (FEM) simulations [11] we have designed zipper cavities appropriate for active material in GaAs and InP based systems. The electric field $\left(E_{y}\right)$ mode profile of a first-order optical mode can bee seen in Fig. 1(b). As is shown in Fig. 1(c) nanobeam width is optimized for both cavity quality factor ( $Q$-factor) to facilitate lasing and optomechanical coupling $\left(g_{\mathrm{OM}}\right)$ to facilitate tuning. Cavity frequency tuning and $g_{\mathrm{OM}}$ are plotted as a function of the gap between nanobeams in Fig. 1(d).
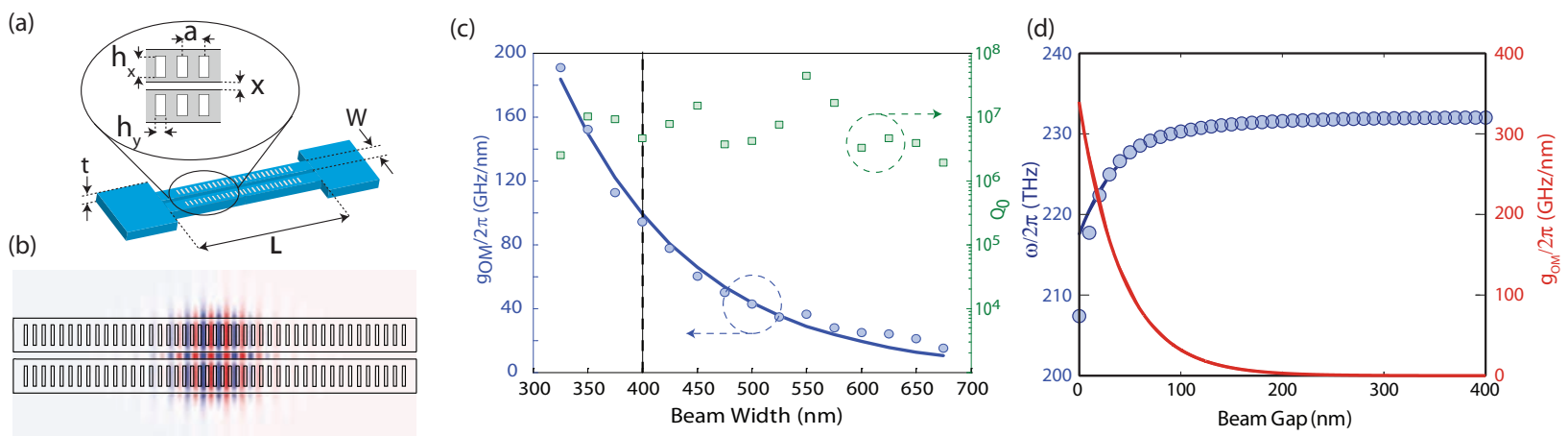

Fig. 1. (a) Zipper cavity doubly clamped nanobeam structure (b) Electric field $\left(E_{y}\right)$ mode profile of first-order defect cavity mode. (c) Optimizing optomechanical coupling $\left(g_{\mathrm{OM}}\right)$ and optical $Q$-factor as a function of nanobeam width. (d) Cavity frequency and $g_{\mathrm{OM}}$ as a function of nanobeam spacing.

In order to study light emission from zipper laser cavity structures initial device work was performed using a semiconductor structure containing a single layer of InAs self-assembled quantum dots [12]. This low-gain active region provides ample light emission from which to perform spectroscopy of the zipper cavity modes, whilst only marginally affecting the measured modal Q-factors (at the level of $5 \times 10^{4}$ ). Zipper cavities are defined via electronbeam lithography and inductively-coupled reactive-ion etching of a $250 \mathrm{~nm}$ thick quantum dot-in-well membrane. Devices are released from the substrate by wet etching of the underneath sacrificial AlGaAs layer [13]. The devices are free space pumped with a pulsed external cavity diode laser at $\lambda=830 \mathrm{~nm}$ (200 ns pulse width, $1 \mu$ s pulse 
period, pump power $P_{p}=317 \mu \mathrm{W}$ ) and free space photoluminescence (PL) is collected via a high numerical aperture objective to a spectrometer. PL measurements show that modes come in bonded and anti-bonded pairs as predicted and measured on passive cavities [11]. First order through third order defect cavity modes can be seen in Fig. 2(a). The modes collectively tune with increase of lattice constant $(a)$ as predicted by simulations. Looking at a bonded mode at very low power ( $10 \mathrm{~ns}$ pulse width, $4 \mu$ s pulse period, pump power $P_{p}=101 \mathrm{nW}$ ) we can estimate an upper bound on its $Q \approx 6000$. Such a mode is quite promising as based on previous work [13] this mode would lase in higher gain material.

(a)

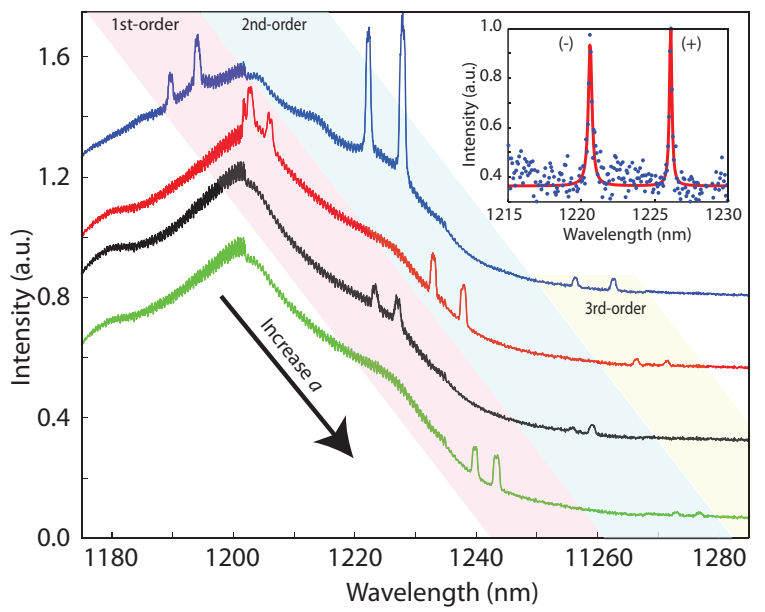

(b)

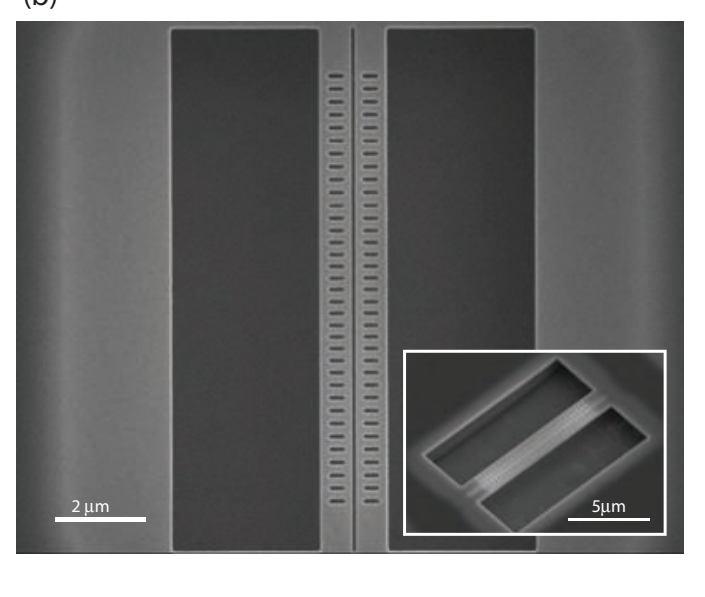

(c)

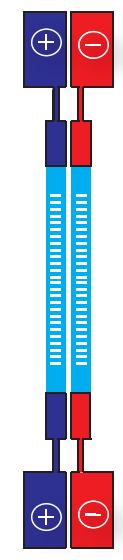

Fig. 2. (a) Photoluminescence spectra of optically pumped Zipper cavities fabricated from GaAs based quantum dot-in-well (DWELL) material. Inset: zoom in of bonded (+) and anti-bonded (-) cavity modes. (b) SEM micrograph top view of DWELL zipper cavity. Inset: Isometric view. (c) Schematic for electrostatic wavelength tuning.

Current work focuses moving designs to higher gain InAsP/GaInAsP quantum well material. Electrostatic mechanism for tuning the gap (see schematic in Fig. 2(c)), similar to work in passive silicon-on-insulator zipper cavities [14], is currently being designed, fabricated, and tested. Up to date experimental and theoretical progress will be reported.

\section{References}

1. Y. Yamamoto and R. E. Slusher, "Optical processes in microcavities," Physics Today 46, 66-73 (1993).

2. R. F. Oulton, V. J. Sorger, T. Zentgraf, R. M. Ma, C. Gladden, L. Dai, G. Bartal, and X. Zhang, "Plasmon lasers at deep subwavelength scale," Nature (London) 461, 629-632 (2009).

3. M. T. Hill, Y. S. Oei, B. Smalbrugge, Y. Zhu, T. D. Vries, P. J. Veldhoven, F. W. M. V. Otten, J. P. Turkiewicz, H. D. Waardt, E. J. Geluk, S. H. Kwon, Y. H. Lee, R. Notzel, and M. K. Smit, "Lasing in metallic-coated nanocavities,” Nature Photonics 1, 589-594 (2007).

4. R. Perahia, T. P. M. Alegre, A. H. Safavi-Naeini, and O. Painter, "Surface-plasmon mode hybridization ins sub-wavelength microdisk lasers," Appl. Phys. Lett. 95, 201114 (2009).

5. L. A. Coldren and S. W. Corzine, Diode Lasers and Photonic Integrated Circuits (John Wiley \& Sons, New York, NY, 1995).

6. E. Bruce, "Tunable Lasers," IEEE Spectrum 39, 35-39 (2002).

7. L. Diehl, B. G. Lee, P. Behroozi, M. Loncar, M. A. Belkin, F. Capasso, T. Aellen, D. Hofstetter, M. Beck, and J. Faist, “Microfluidic tuning of distributed feedback quantum cascade lasers," Opt. Express 14, $11660-11667$ (2006).

8. A. Q. Liu and X. M. Zhang, "A review of MEMS external-cavity tunable lasers," Journal of Micromechanics and Microengineering 17, R1-R13 (2007).

9. M. C. Y. Huang, Y. Zhou, and C. J. Chang-Hasnain, “A nanoelectromechanial tunable laser,” Nature Photonics 2, 180-184 (2004).

10. M. Eichenfield, R. Camacho, J. Chan, K. J. Vahala, and O. Painter, "A picogram- and nanometre-scale photonic-crystal optomechanical cavity," Nature 459, 550-555 (2009).

11. J. Chan, M. Eichenfield, R. Camacho, and O. Painter, "Optical and mechanical design of a "zipper" photonic crystaloptomechanical cavity," Optics Express 17, 3802-3817 (2009).

12. A. Stintz, G. T. Liu, H. Li, L. F. Lester, and K. J. Malloy, "Low-threshold current density 1.3- $\mu$ m InAs quantum-dot lasers with the dots-in-awell (DWELL) structure,” IEEE Photonics Tech. Lett. 12, 591-593 (2000).

13. K. Srinivasan, M. Borselli, O. Painter, A. Stintz, and S. Krishna, "Cavity Q, mode volume, and lasing threshold in small diameter AlGaAs microdisks with embedded quantum dots," Optics Express 14, 1094-1105 (2006).

14. I. W. Frank, P. B. Deotare, M. W. McCutcheon, and M. Loncar, "Dynamically Reconfigurable Photonic Crystal Nanobeam Cavities," http://arxiv.org/abs/0909.2278 [physics.optics] (2009). 